\title{
On the interpretation of expressive adjectives: pragmatics or syntax?
}

\section{Glossa}

a journal of general linguistics

\section{FABIAN BROSS (D)}

\begin{abstract}
It has been observed that sentences containing expressive adjectives modifying a DP can have different readings. In one reading it is the DP referent that is negatively evaluated (called "local interpretation"), while in another reading it is a different DP referent that is evaluated (called "argument hopping"), and in yet another reading it is the whole situation described in the sentence that is being evaluated (called "sentencelevel interpretation"). There are two opposing views on how to capture this ambiguity: pragmatic accounts arguing that the interpretation is governed by contextual factors (via inference) and a recent syntactic account (using an upward-looking version of Agree) arguing that expressive adjectives are scope-taking elements. The goal of the present paper is to clearly identify the different predictions and testing environments necessary to decide which account is more suitable to capture the data. While the results of an experiment designed to test these predictions show a lot of variation, they also suggest that the syntactic constraints proposed by the syntactic account are not responsible for the interpretation of expressive adjectives.
\end{abstract}

\section{] $\mathrm{u}[$ ubiquity press}

SQUIB

CORRESPONDING AUTHOR:

\section{Fabian Bross}

University of Stuttgart, Keplerstr. 17, 70174 Stuttgart, DE

fabian.bross@ling.uni-stuttgart.de

\section{KEYWORDS:}

expressivity; expressive adjectives; scope; pragmatics; syntax

TO CITE THIS ARTICLE: Bross, Fabian. 2021. On the interpretation of expressive adjectives: pragmatics or syntax? Glossa: a journal of general linguistics 6(1): 71. 1-13. DOI: https://doi. org/10.5334/gjgl.1214 


\section{Introduction}

Sentences containing expressive adjectives (EAs) like damn can have different interpretations, cf. example (1) adapted from Gutzmann (2019). Intuitively, the sentence can be used by the speaker to express her/his negative attitude towards the sausage, the dog, or even the whole situation described in the sentence which receives a negative evaluation (Potts 2005; Frazier, Dillon \& Clifton 2015; Gutzmann 2019).

(1) The dog ate the damn sausage.
a. $:($ the sausage)
b. $\otimes($ the dog)
c. $\odot$ (the dog ate the sausage)

For illustrative purposes Gutzmann (2019) uses an easy-to-capture smiley notation which I will adopt here. The narrow reading in which it is only the DP the sausage which is evaluated is shown in (1a). The reading in which it is the DP which is not modified by the expressive and yet is negatively evaluated is shown in (1b).

This reading is quite surprising because the adjective is interpreted on a constituent different from the one it is overtly modifying. Cases like these are called "argument hopping" by Gutzmann (2019). Here, argument hopping is, linearly speaking, to the left (from an adjective modifying the object to the subject). This is accordingly called "left hopping". Cases in which a DP referent is negatively evaluated although the EA modifies a preceding DP are correspondingly called "right hopping". The wide reading, also called "sentence-level interpretation", is illustrated in (1c). With the sentence-level interpretation it is the whole situation described by the sentence which is evaluated. Such cases, in which the scope of the evaluation applies to a larger constituent are called "argument extension" by Gutzmann. Though multiple interpretations are possible, especially when embedded clauses are additionally considered, the current study mainly focus on the following possibilities: ${ }^{1}$

Root clauses:

a. Argument hopping (left or right): The referent of a DP different from the damn DP can receive a negative evaluation.

b. Argument extension: A root clause can receive a sentence-level interpretation when it contains a damn DP.

Complex clauses with damn only in the embedded clause:

a. Matrix-subject interpretation (a special case of left hopping crossing a CPboundary): The referent of the matrix-subject DP is negatively evaluated, even though the damn DP is in the embedded clause.

b. Matrix-clause interpretation (a special case of argument extension crossing a CP-boundary): The whole situation expressed by the complex clause is negatively evaluated, even though the damn DP is in the embedded clause.

Concrete examples of each possibility will be presented later. There are two opposing views in the literature that capture the intuition that sentences involving an EA can have different readings: pragmatic accounts (Potts 2007; Frazier, Dillon \& Clifton 2015) and a recent syntactic account (Gutzmann 2019). In the following, I will briefly review these two accounts and discuss some open issues concerning the empirical research conducted so far (Section 2). In Section 3, an experiment is presented which tests the different predictions of the two accounts. In Section 4, I conclude.

\section{Pragmatics or syntax? Two accounts of EAs 2.1 Frazier, Dillon \& Clifton's pragmatic approach}

Frazier, Dillon \& Clifton (2015) suggest, based on Potts (2005; 2007), that the interpretation of an EA comes about via inference. They assume that not-at-issue content, in general, constitutes

1 There are many more theoretically possible cases (e.g., hopping from a matrix into an embedded clause) and, in addition, Gutzmann (2019) also discusses cases of clause-external EAs like Damn! The dog ate the sausages. However, for reasons of simplicity I will not discuss these cases here. 
a quasi-independent speech-act. This assumption is called the "speech-act hypothesis". This hypothesis states that

an expressive like damn constitutes a speech-act separate from the speech-act of the at-issue content conveyed by the rest of the sentence (Potts 2005; 2007) and permits the expressive to be interpreted with respect to portions of the utterance (including the entire utterance) other than its syntactic sister. (Frazier, Dillon \& Clifton 2015: 299)

Additionally, they assume that the interpretation of an expressive also depends on the presence of an agentive causer in the sentence. The likelihood of a sentence containing an EA receiving an interpretation in which the negative attitude is conveyed towards the subject is higher when the subject expression refers to an individual which can be held responsible-irrespective of the exact position of the EA. Thus, the sentence in (4a) is according to this view-called the "culprit hypothesis"-more likely to receive a subject interpretation (i.e., a left-hopping reading) than the sentence in (4b) (examples from Frazier, Dillon \& Clifton 2015).

a. The dog is on the damn couch.

b. The holiday is on the damn weekend.

Frazier, Dillon \& Clifton (2015) indeed present experimental evidence in favor of the speechact and the culprit hypothesis. First, they show that participants (forty-eight undergraduate students) interpreted sentences containing an EA as expressing a negative attitude towards the whole situation when the expressive modified a subject or object DP. Second, they show that participants report more subject interpretations with causal items than with non-causal ones. In the following, I will refer to this account as PA (for "pragmatic account").

\subsection{Gutzmann's syntactic approach}

Gutzmann (2019) rejects PA. He claims that a matrix-clause interpretation is not possible when an EA appears in an embedded clause. Consider the sentence in (5) from Gutzmann (2019: 100).

(5) Peter said that the dog ate the damn cake.

PA predicts that the sentence should have (at least) four possible readings, illustrated in (6). However, the sentence-level interpretation is, according to Gutzmann (2019), not possible, as indicated.
a. $\odot$ (the cake)
b. $:($ the dog)
c. $\odot$ (the dog ate the cake)
d. $\&$ (Peter said that the dog ate the cake) 


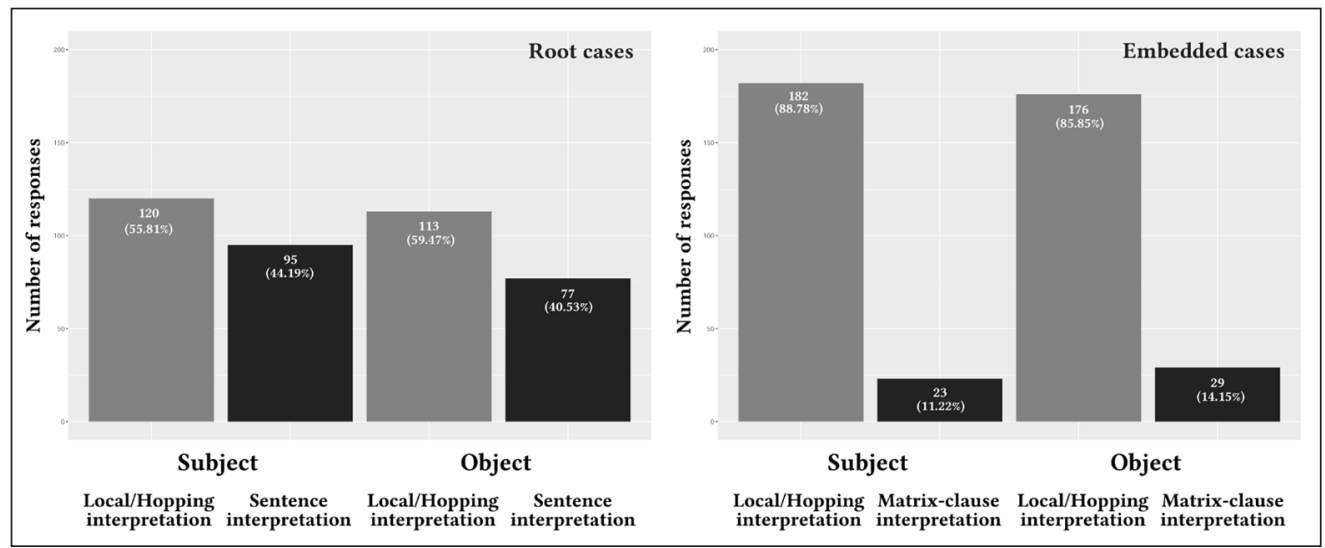

him, only possible because the sentence-level interpretation is still active. This, in turn, makes a subject interpretation possible. However, this is not a syntactic mechanism, but an implicature. Thus, to arrive at the subject interpretation in a case in which the EA is attached to the object, one additional step is involved, as represented in (7) (based on Gutzmann 2019: 107). This means that argument extension is possible under certain syntactic environments. Argumenthopping readings, in contrast, are only available as pragmatic derivatives from argument extension.

$$
\begin{aligned}
& \text { The dog ate the damn sausages. } \\
& \approx \otimes \text { (the dog ate the sausages) } \\
& +>\text { The speaker is angry about the dog. }
\end{aligned}
$$

(argument extension) (implicature)

To show this, Gutzmann (2019) argues that in cases in which the sentence-level interpretation is semantically disfavored the subject interpretation also does not arise. For this purpose, he uses examples with a sentential adverbial like fortunately (8). A subject interpretation in (8), i.e., an interpretation where the speaker is happy about the eating event and at the same time dislikes the dog, indeed seems hardly possible.

Fortunately, the dog (finally) ate the damn sausages.

To model the restrictions presented, Gutzmann (2019) uses an upward-looking version of Agree (Zeijlstra 2012). The EA itself carries an uninterpretable expressivity feature Ex. What is interpreted is a syntactic expressivity feature that needs to be higher up in the structure in a c-command relation with the EA (within a single $\mathrm{CP}$ ). The simplest case is an EA modifying a DP receiving a local interpretation. In this case, the interpretable feature is located in the head of the DP, as illustrated in the trees in (9). (9a) shows the starting configuration. The uninterpretable expressivity feature on damn represents the probe looking upwards for a c-commanding goal: the interpretable expressivity feature located in the D head. In a second step (9b) feature valuation takes place and the uninterpretable feature on damn can be deleted.

a.

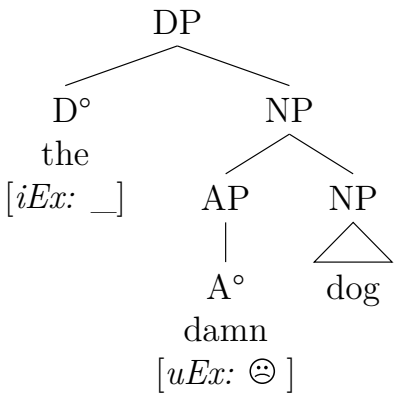

b.

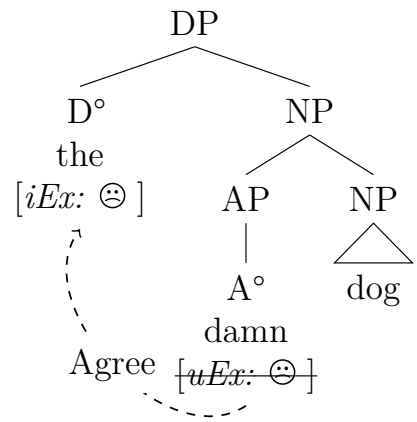

This model immediately explains why argument extension is possible, i.e., why it is, for example, possible that an EA modifying a subject DP can lead to a sentence-level reading. In

Figure 1 Results from Gutzmann's study. The rootclause condition is shown on the left and the embeddedclause condition on the right. The labels "subject" and "object" indicate the position of the EA. 
this case, the $i E x$ feature is located in $\mathrm{C}^{\circ}$, c-commanding the expressive, as illustrated in the simplified tree in (10). But what about left and right hopping? According to Gutzmann (2019: 112), both cases are ruled out in this model as "the interpretable and uninterpretable features do not stand in a c-command relation, irrespective of whether the hopping is supposed to be to left [...] or right [...]". This is also visible in the tree in (10). I will refer to this model as ULAM ("upward-looking Agree model").

(10)

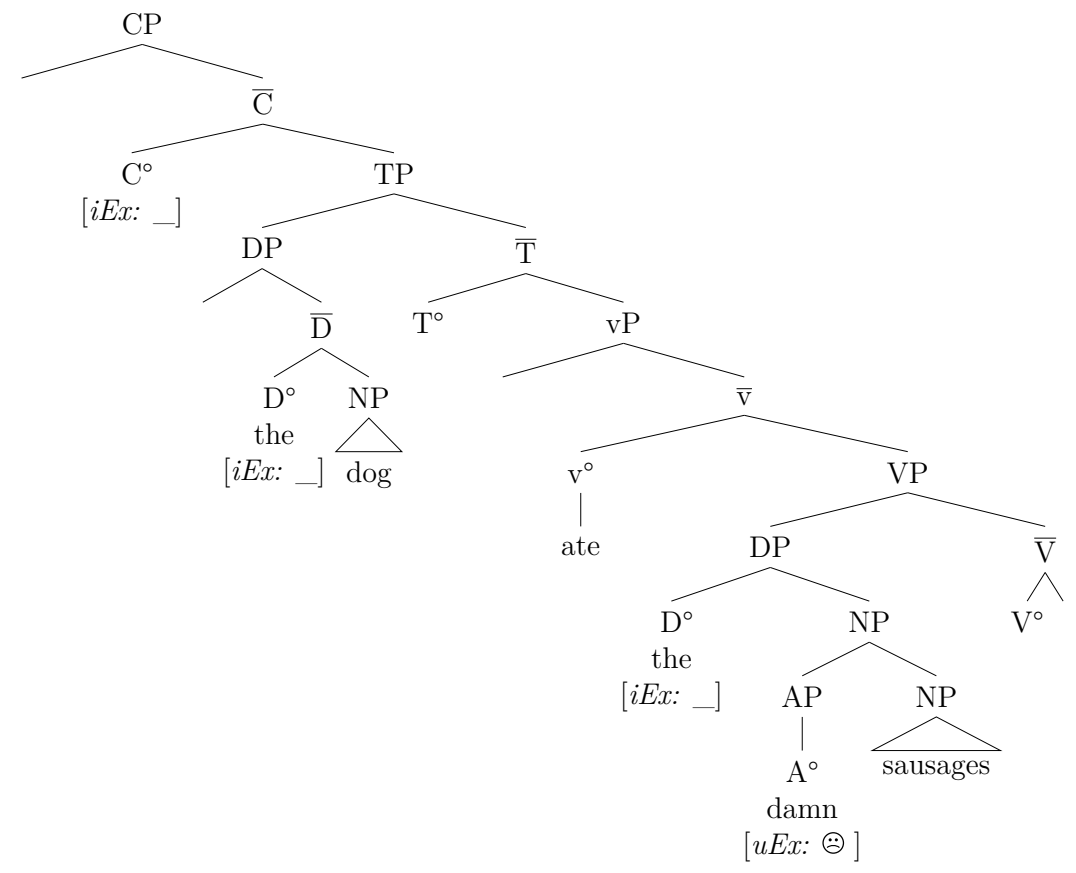

\subsection{Open issues}

In his experiment, Gutzmann asked participants to rate what the most probable interpretation of a sentence was (similar to Frazier, Dillon \& Clifton 2015). What is of interest to evaluate ULAM, however, is not the most probable interpretation, but rather which interpretations are possible and which are not. The main goal of his experiment was to show that there is a CPblocking effect. Even if participants were asked for the most probable interpretation, in over ten percent of the cases when the expressive was modifying the embedded subject and in nearly fifteen percent of the cases when the expressive was modifying the embedded object, the matrix-clause interpretation was chosen (cf. the right part of Figure 1). What this tells us is that argument extension from an embedded clause into a matrix clause is disfavored when presented without context, but the conclusion that it is syntactically blocked seems to be too strong. It would have been interesting to see whether participants in general thought that this reading was (im)possible.

Additionally, the experiment only tested the matrix-clause interpretation and not argument hopping (including the matrix-subject interpretation). However, all four cases are of interest as ULAM and PA make different predictions about their availability. These different predictions were tested in an experiment discussed in the next section.

\section{Experiment}

The experiment discussed here was designed to test the different predictions PA and ULAM make concerning the availability of non-local readings: the possibility of left hopping in root clauses starting with fortunately, the possibility of right hopping in root clauses starting with fortunately, and the question of whether CP-barriers exist (i.e., if a matrix-clause reading/ matrix-subject reading from an embedded clause containing an EA is possible). Participants in this study were presented with context-sentence pairs and were asked whether they would allow or disallow a target sentence with a certain interpretation in a specific context. The experiment was conducted in German. 
The general reasoning behind this sentence-to-context matching task was the following: The context made clear that the speaker has a positive attitude towards all referents appearing in a target sentence except for one. In the target sentence, the EA modified a DP whose referent was clearly established to refer to a positively evaluated referent. Participants then had to choose if it was possible to them that the sentence could be used in that context. If this was not possible the participants only allowed for the local reading, and if this was possible the participants allowed for argument hopping/extension.

\subsection{Materials and procedure}

Before presenting the materials, some preliminary thoughts on the make-up of the examples to be used are in order because examples making different predictions concerning PA and ULAM are needed.

When it comes to root cases, different predictions emerge if the sentence-level interpretation is made implausible, as shown in (11). PA predicts left hopping to be possible if the non-damn DP is an agentive causer. ULAM, however, predicts left hopping cases to be impossible for syntactic reasons.

Fortunately, the $\mathrm{X}$ verbed the damn $\mathrm{Y}$.

left hopping: $:$ (the $\mathrm{X}$ )

PA: $\checkmark$ ULAM: $x$

The reverse case is shown in (12). PA predicts right hopping to be theoretically possible, especially if $\mathrm{X}$ is not a potential causer. ULAM, again, predicts hopping to be syntactically blocked as the sentence-level interpretation is semantically disfavored.

Fortunately, the damn $\mathrm{X}$ verbed the $\mathrm{Y}$.

right hopping: $:($ the $\mathrm{Y}$ )

PA: $\checkmark$ ULAM: $x$

Finally, consider embedded cases. While there are theoretically many different possible interpretations, the relevant ones are the matrix-clause interpretation and the matrix-subject interpretation. PA predicts the matrix-clause interpretation to be possible, while ULAM predicts it to be impossible because of an intervening CP-barrier. Similarly, the matrix-subject interpretation should be possible according to PA and blocked according to ULAM, again because of an intervening CP-barrier. This is shown in (13).

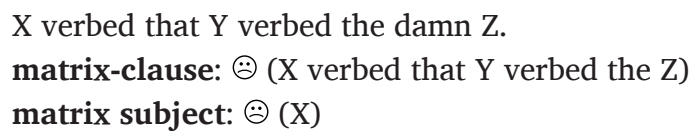

PA: $\checkmark$ ULAM: $x$

PA: $\checkmark$ ULAM: $x$

The sentence structures of the examples used in the experiment slightly deviate from the simple (abstract) examples above to create plausible scenarios in which the desired evaluations might become possible.

Condition 1 (left hopping): A first requirement for a plausible left-hopping case is that the subject needs to be an agentive causer. This, in general, is not problematic. However, an interference arises because of the evaluative sentential adverb: Positively evaluating the whole proposition by using an adverb like fortunately will make a negative evaluation of the subject as the plausible causer of the described action unlikely. One possibility to nevertheless create a plausible scenario can be seen in examples in which the speaker positively evaluates the subject referent's disappearance. This was done in this condition, as exemplarily shown in (14). All examples in the left-hopping condition were preceded by contexts making clear that the speaker has a positive attitude towards the damn DP referent and a negative attitude towards the non-damn DP referent. The target sentence was followed by two options (in random order without numbers). One option stated that it is possible to use the sentence in this context to only negatively evaluate the non-damn DP referent while at the same time not to negatively evaluate the damn DP referent. The other option was that the target sentence is not suitable in the context.

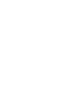


Jasmin hates ants and is scared of them. In her garden she built an ant trap consisting of a hole filled with honey. She is very proud of her construction. Suddenly, she is surrounded by ants and gets scared. But then she realized that the ants are not interested in her, but in the honey in the hole. She is happy about this and says to herself:

Fortunately, the ants will jump into the damn trap.

Option 1: I think it is possible for Jasmin to utter this sentence to negatively evaluate the ants and at the same time NOT to negatively evaluate the trap.

Option 2: I think it is not possible to use this sentence in this context.

PA predicts that participants choose option 1 as left hopping should be possible. While the target of the negative evaluation is clear from the context, if hopping does not exist the sentence should not be an available option as it would contain an EA which can only contribute a local reading ruled out by context. In this case, option 2 should be chosen, as predicted by ULAM.

Condition 2 (right hopping): For the right-hopping cases another set of examples were created with verbs describing the prevention of an action with a non-conscious inanimate subject. An example sentence, enriched with a context, is shown in (15).

Patrick works as a programmer and has developed a computer program of which he is very proud. The program was installed on the computers of an important customer who recently was the victim of a hacker attack. An investigation of the attack revealed that Patrick's program ensured that the viruses the hackers wanted to load on the customer's computers were blocked. Patrick reports the events to a colleague:

Fortunately, the damn computer program blocked the viruses.

Option 1: I think it is possible for Patrick to utter this sentence to negatively evaluate the viruses and at the same time to NOT negatively evaluate the computer program.

Option 2: I think it is not possible to use this sentence in this context.

Again, PA predicts hopping to be generally possible and we would thus expect participants to choose option 1. If right hopping was not possible, as predicted by ULAM, there would be a meaning clash between the EA in the clause which should only have a local interpretation and the context which says that Patrick is very proud of his program. Thus, ULAM predicts that participants should choose option 2 .

Condition 3 (CP barriers): The third condition consisted of matrix clauses followed by an embedded clause containing an EA. The goal of this condition was to determine if the matrixclause interpretation and/or the matrix-subject interpretation are possible with the local interpretation being not available at the same time. Contexts were chosen in a way which made clear that the matrix event was somehow undesirable and that the local interpretation was not active at the same time. An example is given in (16). In this condition, the context and the target sentence were followed by three options. First, participants had the option that it is possible that it is the matrix subject which the speaker negatively evaluates, but not the DP modified by the EA nor the embedded proposition. The second option targeted the matrix-clause interpretation in a similar way and finally, the third option stated that the sentence is not felicitous in the context.

Jean bought a new car which he really likes. He is super happy. Originally, the money he spent on the car was meant for a new computer he needs for school, but he doesn't care about that. After he learns that his brother Christian snitched on him and told his father about him buying the car he tells his buddy about the events:

My brother Bob told my dad that I bought the damn car.

Option 1: I think it is possible for Jean to utter this sentence to negatively evaluate his brother Bob while he does NEITHER negatively evaluate the car NOR the fact the he bought the car at the same time. 
Option 2: I think it is possible for Jean to utter this sentence to negatively evaluate the fact that his brother Bob told his dad that he bought the car while he does NEITHER negatively evaluate the car NOR the fact the he bought the car at the same time.

Option 3: I think it is not possible to use this sentence in this context.

PA predicts that option 1 and option 2 are available, i.e., that it is possible that examples like the one in (16) have an interpretation in which the speaker negatively evaluates the matrix subject while the EA has no local/embedded clause interpretation at the same time, and it has an interpretation in which it is the act of reporting the embedded proposition which is negatively evaluated while the local/embedded clause interpretation are not active. ULAM, in contrast, predicts that neither of these interpretations should be available. Note that while participants had three choices here (matrix-clause reading possible, matrix-subject reading possible, and/or only the local reading available) this condition can be broken down into two binary choices for the analysis: Either the matrix-clause interpretation was judged to be possible or not, and either the matrix-subject interpretation was judged to be possible or not.

Each participant was presented with four items enriched with contexts in each condition. Thus, each participant was presented with twelve context-sentence pairs. Additionally, twelve filler items were added without EAs, but also with contexts. The filler items were root clauses containing an evaluative adjective (half of them negative ones like unfortunately, half of them positive ones like fortunately). The questions provided served as control questions testing whether participants paid attention to the task. Additionally, the filler items were much easier to process than the control items and thus participants were not required to keep the same amount of concentration during the whole study. An example is shown in (17).

Context: Maria inherited a very expensive watch from her grandfather. However, she lost the watch last week at a party. Her brother Karl tells his buddy about the event:

Unfortunately, Maria lost the watch.

Option 1: I think it is possible that Karl utters this sentence to negatively evaluate the fact that Maria lost the watch.

Option 2: I think it is possible that Karl utters this sentence to positively evaluate the fact that Maria lost the watch.

Option 3: I think it is not possible to use this sentence in this context.

All materials can be found in the supplementary files of this paper. A Latin-square design was chosen with three lists. As each participant was presented with twelve critical items a total of thirty-six items were created. Participants were randomly assigned to one of the lists. The presentation of stimuli was randomized for each participant. Additionally, the order of presentation of the available options was randomized. The experiment was conducted as an online survey using Google Forms. For each list one Google Forms document was created. Participants entered the experiment through a specially built website which randomly assigned them to one of the lists.

\subsection{Participants}

The study was advertised through a university-internal mailing list. The subscribers of this list were students of German. The potential participants were told that the study was about their sprachgefühl and would require them to closely pay attention to the task. Eighty-three students participated in the study. The responses of participants wrongly answering more than two control questions added as fillers were not considered in the final analysis. Based on this criterion the responses from ten participants were removed. The reason for this procedure was that the study required the subjects to really pay attention to the task. ${ }^{2}$ The remaining seventy- 
three participants had a mean age of $26.55(S D=10.90)$, fifty-nine of them self-identified as females and one of them as non-binary. All participants were self-reported native speakers of German and none of them reported any language disorders. All participated voluntarily without any payment.

\subsection{Results}

Overview of the data: Participants allowed a left-hopping interpretation in exactly $50 \%$ of the cases. Nearly the same results were obtained for right hopping with $54.45 \%$ of the judgments allowing for right hopping. An overview of the data is shown in Figure 2. One question is whether there are two different grammars, i.e., one question is whether there were two groups of participants with one group allowing for argument hopping and the other group disallowing it. While there indeed were some participants who disallowed hopping completely, this is not the general pattern: Only fifteen participants (i.e., 20.55\%) did not allow left hopping at all and fifteen participants did not allow right hopping at all. Subjects disallowing left and right hopping were not necessarily the same individuals. Only seven participants (i.e., 9.59\%) disallowed both left and right hopping (and of these participants only three additionally disallowed for the matrix-clause/matrix-subject interpretation). This means that $90.41 \%$ of the participants did indeed allow for argument hopping (in root clauses) in at least one case. The behavior of the individual participants is shown in Figure 3.

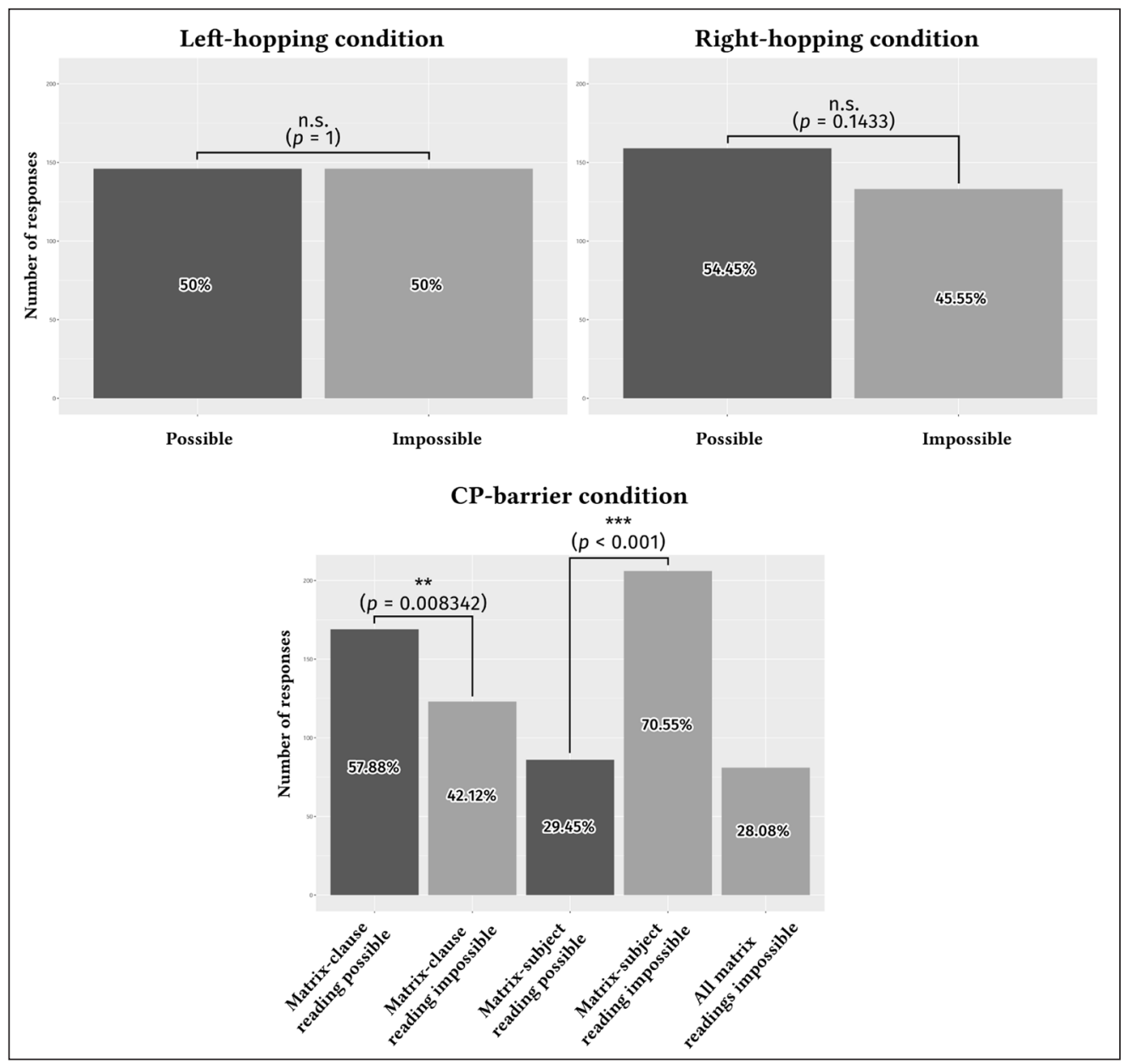

The target stimuli in the CP-barrier condition were judged to be generally impossible in $28.08 \%$ of the cases. $57.88 \%$ of the examples were judged to have the matrix-level interpretation and the matrix-subject interpretation was available in $29.45 \%$ of the cases. Only nine participants disallowed the matrix-clause interpretation altogether, but twenty-nine completely disallowed the matrix-subject interpretation in all cases (or, put the other way around: $87.67 \%$ allowed the matrix-clause interpretation at least in one case, but only $60.27 \%$ allowed the matrix-subject interpretation).
Figure 2 Results of the study (with "n.s." meaning nonsignificant). 


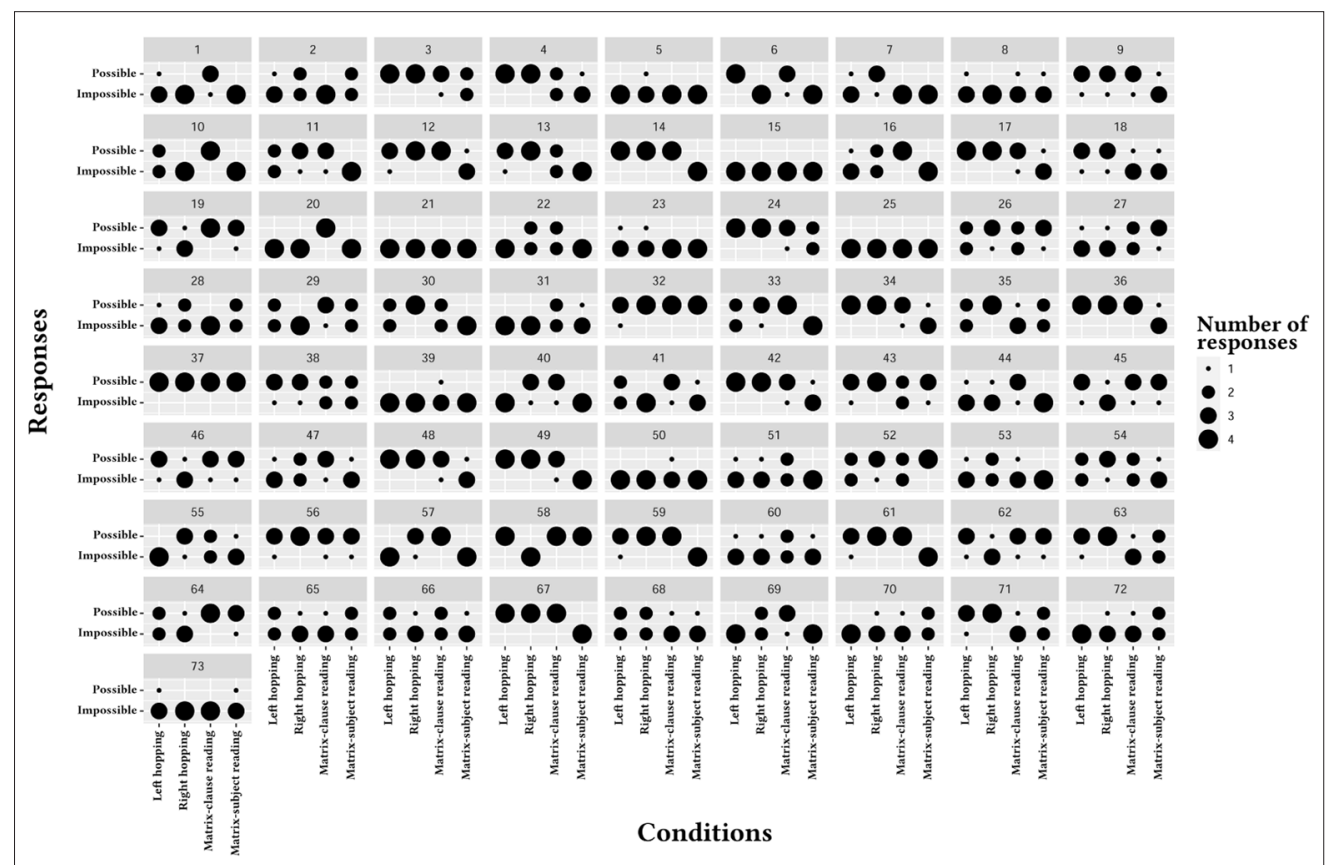

Inferential statistics: For the left and right hopping conditions the outcome was binary (a sentence was either judged to be possible in a context or not). This was similar in the CP-barrier condition considering that participants could rate the matrix-clause interpretation as being possible or not and, in addition, could rate the matrix-subject interpretation as being possible or not. A binomial generalized linear mixed model was fitted using the lme4 package (Bates et al. 2015) in R ( R Core Team 2020) predicting the responses by condition (i.e., left hopping, right hopping, matrixsubject, and matrix-clause reading) with random intercepts for participants and items and random slopes for participants. The different conditions were contrasted using the lsmeans package (Lenth 2016). The results of the model are shown in Table 1 and the results of the pairwise comparison in Table 2. There were three statistically significant contrasts: The matrix-subject interpretation was significantly less often chosen compared to the left- and compared to the right-hopping option, and the matrix clause-interpretation was significantly more often chosen compared to the matrix-subject option. Note that besides comparing the different conditions, it is also possible to compute $p$ values by comparing the binary outcomes inside each condition (whether a given interpretation was judged to be possible or not). For this purpose, simple binomial tests were used revealing a statistically significant result for the matrix-subject and matrix-clause interpretation. The results of these tests are depicted in Figure 2. Note that in the argument-hopping conditions it seems as if participants have simply guessed or did not pay enough attention to the task. There are three reasons speaking against this. First, the results are much clearer in the matrix-clause conditions (which are also more complex). Second, participants did perform well on fillers. Finally, participants were consistent in their judgments; this is evidenced by the fact that the judgments of the items in each condition are correlated, i.e., they show internal consistency. As a measure of internal consistency, the Kuder and Richardson Formula 20 (KR-20) (Kuder \& Richardson 1937) and Cronbach's alpha (Cronbach 1951) was used. Both measure the reliability of the items. All items in each condition were constructed to measure the same thing. If the items in one condition indeed measure the same thing one would expect that each participant is consistent in her/his judgments of these items (and not, for example, randomly judging left hopping to be possible in half of the cases and to be impossible in the other half). If this is the case the judgments of the items (in a specific condition for a specific participant) should be correlated.

\begin{tabular}{lllll}
\hline & Estimate & Std. Error & $z$ value & $\operatorname{Pr}(>|z|)$ \\
\hline (Intercept) & -0.02612 & 0.31479 & -0.083 & 0.93386 \\
\hline conditionmatrixclause & -0.39533 & 0.41066 & -0.963 & 0.33570 \\
\hline conditionmatrixsubject & -1.29140 & 0.43531 & -2.967 & 0.00301 \\
\hline conditionrighthopping & -0.45417 & 0.38180 & -1.190 & 0.23422 \\
\hline
\end{tabular}

general linguistics

DOI: 10.5334 /gjgl.1214

Figure 3 By-participant decisions. The plot shows the behavior of all participants. Each participant rated four items in each condition. Participant 1, for example, disallowed the matrix-subject reading in all four cases. The same is true for the righthopping items. From the four left-hopping items this participant was presented with, one was judged to have the left hopping reading and three not. The opposite is true for participant 1's judgments for the matrix-clause items.

Table 1 Results of the binomial model. 


\begin{tabular}{lllll}
\hline Contrast & Estimate & SE & z ratio & p value \\
\hline lefthopping - matrixclause & -0.3953 & 0.411 & -0.963 & $<0.7707$ \\
\hline lefthopping - matrixsubject & -1.2914 & 0.435 & -2.967 & $<0.0159$ \\
\hline lefthopping - righthopping & -0.4542 & 0.382 & -1.190 & $<0.6335$ \\
\hline matrixclause - matrixsubject & -1.6867 & 0.291 & -5.802 & $<0.0001$ \\
\hline matrixclause - righthopping & -0.0588 & 0.492 & -0.120 & $<0.9994$ \\
\hline matrixsubject - righthopping & -1.7456 & 0.532 & -3.280 & $<0.0057$ \\
\hline
\end{tabular}

Bross

Glossa: a journal of

general linguistics

DOI: 10.5334 /gjgl.1214

Table 2 Pairwise comparisons of the different conditions with Ismeans.
KR-20 and Cronbach's alpha are similar measures, but KR-20 measures the test score reliability for binary data. For the interpretation of both the rule of thumb for Cronbach's alpha can be used as both measures range from 0 to 1 :

- Values between 0.9 and 1.0 show extremely high correlations (i.e., a high reliability of participants over items holding decisions fixed).

- Values between 0.7 and 0.9 still show a strong correlation.

- Values between 0.6 and 0.7 are acceptable and

- values below 0.6 show poor correlation.

Note that KR-20 consistently underestimates reliability and thus produces slightly lower values (Kuder \& Richardson 1937). The items of the left-hopping condition revealed a KR-20 of $\rho=$ 0.71 and the ones in the right-hopping condition of $\rho=0.80$. For the CP-barrier condition a Cronbach's alpha was calculated revealing a $\rho=0.64$. If the questions whether the matrixsubject and the matrix-clause interpretation are possible are looked at in isolation, a KR-20 can also be calculated. Only looking at whether the matrix-subject interpretation was possible or not the KR-20 formula revealed a value of $\rho=0.58$ (which is a little below the threshold) and for the matrix-clause interpretation a KR-20 of $\rho=0.61$ was calculated. In sum, these values are in an acceptable range indicating that responses were not random, but that participants, in general, consistently judged the items in each condition and thus did not guess or make random judgments.

\subsection{Discussion}

Although the results are not clear-cut, they do argue against the syntactic constraints proposed by ULAM. Argument hopping in root clauses was available in approximately $50 \%$ of the cases even though ULAM predicts hopping to be syntactically impossible. Note that these results are similar to those presented in Gutzmann (2019) where there also was a lot of variation with root clauses. For the embedded clauses, the matrix clause reading was available in nearly $60 \%$ of the cases, while the matrix-subject reading was only available in approximately $30 \%$ of the cases, also suggesting that there is no strict syntactic constraint ruling out these interpretations.

On first sight, these results are also not as clear-cut as predicted by PA. The interpretation of EAs seems to be extremely flexible on the one hand and subjective on the other. The reason for this may be that there are more pragmatic factors playing a role in the interpretation of EAs than previously thought. As noted by an anonymous reviewer, PA does not predict full acrossthe-board acceptability of all readings because people's judgments are probably influenced by a lot of pragmatic considerations. Pragmatic accounts thus predict there to be variation in the judgment of the availability of non-local readings. On the other hand, it can, of course, also not be precluded that the interpretation of EAs is governed by yet unknown syntactic constraints not covered by ULAM.

One limitation of the study presented is that it was only students of German who participated which might have had an effect on their judgments (it might, for example, be that some of them were more conservative in their judgments than the general population). A more severe problem is the high variability of judgments in the argument-hopping conditions. As discussed, this has probably nothing to do with the fact that participants did not pay enough attention to the task as only those participants were considered who performed well on the control items 
added as fillers. Additionally, the fact that the results are more clear-cut in the embeddedclause condition suggests that participants did not simply guess. This view is also supported by the reliability measures presented.

\section{Conclusion}

This squib was concerned with two competing accounts of the interpretation of expressive adjectives, namely pragmatic accounts arguing that it is mainly contextual factors driving their interpretation and a recent syntactic account using an upward-looking version of Agree. I identified the testing environments necessary to decide which of these accounts is more suitable to capture the data and put the different predictions the accounts make to test. More precisely, the upwards-looking Agree model predicts that expressive adjectives in root clauses containing positively evaluating sentential adverbs only allow a local interpretation, i.e., argument hopping should not be possible. Additionally, this model predicts that an expressive adjective located in an embedded clause cannot be used to negatively evaluate a referent or a proposition expressed in the matrix clause. According to pragmatic accounts, in contrast, these readings should, in principle, exist. The results of the experiment presented show that participants' judgments varied a lot. Argument hopping was judged to be possible in approximately $50 \%$ of the cases, while argument extension from an embedded into a matrix clause was possible in approximately $60 \%$ of the cases and argument hopping into a matrix clause in approximately $30 \%$ of the cases. While these results are not clear-cut, they show that the syntactic constraints proposed so far seem not to guide the interpretation of expressive adjectives. This, however, does not mean that there are generally no syntactic mechanisms involved. Similarly, it is possible that there are pragmatic mechanisms at work which have been overlooked so far that have contributed to the high variability of judgments. Nevertheless, the discussion presented in this squib should help future researchers in carefully designing empirical tasks to further investigate this issue.

\section{Additional files}

The additional files for this article can be found as follows:

- Stimuli.pdf. A file containing all stimuli used in the experiment (in German and English). DOI: https://doi.org/10.5334/gjgl.1214.s1

- The file data.csv. A file containing the data. DOI: https://doi.org/10.5334/gigl.1214.s2

- A file called statistics.r. A file containing the R script to reproduce the statistical results. DOI: https://doi.org/10.5334/gjgl.1214.s3

\section{Competing interests}

The author has no competing interests to declare.

\section{Author affiliation}

Fabian Bross iD orcid.org/0000-0003-3843-3568

University of Stuttgart, Keplerstr. 17, 70174 Stuttgart, DE

\section{References}

Bates, Douglas, Martin Mächler, Ben Bolker \& Steve Walker. 2015. Fitting linear mixed-effects models using lme4. Journal of Statistical Software 67(1). 1-48. DOI: https://doi.org/10.18637/jss.v067.i01

Cronbach, Lee J. 1951. Coefficient alpha and the internal structure of tests. Psychometrika 16(3). 297334. DOI: https://doi.org/10.1007/BF02310555

Frazier, Lyn, Brian Dillon \& Charles Clifton. 2015. A note on interpreting damn expressives: transferring the blame. Language and Cognition 7(2). 291-304. DOI: https://doi.org/10.1017/langcog.2014.31

Gutzmann, Daniel. 2019. The grammar of expressivity. Oxford: Oxford University Press. DOI: https://doi. org/10.1093/oso/9780198812128.001.0001

Kuder, G. Frederic \& Marion W. Richardson. 1937. The theory of the estimation of test reliability. Psychometrika 2(3). 151-160. DOI: https://doi.org/10.1007/BF02288391 
Lenth, Russell V. 2016. Least-squares means: the R package lsmeans. Journal of Statistical Software 69(1). 1-33. DOI: https://doi.org/10.18637/jss.v069.i01

Potts, Christopher. 2005. The logic of conventional implicatures. Oxford: Oxford University Press. DOI: https://doi.org/10.1093/acprof:oso/9780199273829.001.0001

Potts, Christopher. 2007. The expressive dimension. Theoretical Linguistics 33(2). 165-198. DOI: https:// doi.org/10.1515/TL.2007.011

R Core Team. 2020. R: A Language and Environment for Statistical Computing. Vienna, Austria: $\mathrm{R}$ Foundation for Statistical Computing. https://www.Rproject.org/.

Zeijlstra, Hedde. 2012. There is only one way to agree. The Linguistic Review 29(3). 491-539. DOI: https:// doi.org/10.1515/tlr-2012-0017
Glossa: a journal of general linguistics

DOI: 10.5334 /gjgl.1214
TO CITE THIS ARTICLE: Bross, Fabian. 2021. On the interpretation of expressive adjectives: pragmatics or syntax? Glossa: a journal of general linguistics 6(1): 71. 1-13. DOI: https://doi. org/10.5334/gjgl.1214

Submitted: 06 February 2020 Accepted: 12 March 2021 Published: 25 May 2021

COPYRIGHT:

(c) 2021 The Author(s). This is an open-access article distributed under the terms of the Creative Commons Attribution 4.0 International License (CC-BY 4.0), which permits unrestricted use, distribution, and reproduction in any medium, provided the original author and source are credited. See http:// creativecommons.org/ licenses/by/4.0/.

Glossa: a journal of general linguistics is a peer-reviewed open access journal published by Ubiquity Press. 